\title{
Selection on Codon Usage for Error Minimization at the Protein Level
}

\author{
Marco Archetti \\ Département de Biologie, Section Ecologie et Evolution, Université de Fribourg, Chemin du Musée 10, 1700, Fribourg, Switzerland
}

Received: 30 September 2003 / Accepted: 12 April 2004 [Reviewing Editor: Dr. Massimo Di Giulio]

\begin{abstract}
Given the structure of the genetic code, synonymous codons differ in their capacity to minimize the effects of errors due to mutation or mistranslation. I suggest that this may lead, in protein-coding genes, to a preference for codons that minimize the impact of errors at the protein level. I develop a theoretical measure of error minimization for each codon, based on amino acid similarity. This measure is used to calculate the degree of error minimization for 82 genes of Drosophila melanogaster and 432 rodent genes and to study its relationship with CG content, the degree of codon usage bias, and the rate of nucleotide substitution. I show that (i) Drosophila and rodent genes tend to prefer codons that minimize errors; (ii) this cannot be merely the effect of mutation bias; (iii) the degree of error minimization is correlated with the degree of codon usage bias; (iv) the amino acids that contribute more to codon usage bias are the ones for which synonymous codons differ more in the capacity to minimize errors; and (v) the degree of error minimization is correlated with the rate of nonsynonymous substitution. These results suggest that natural selection for error minimization at the protein level plays a role in the evolution of coding sequences in Drosophila and rodents.
\end{abstract}

Key words: Genetic code - Error minimization Codon usage - Nucleotide substitution Drosophila melanogaster — Rodents - Amino acid similarity

Correspondence to: Marco Archetti; email: marco.archetti@ unifr.ch

\section{Introduction}

Studies on the origin of the genetic code have shown that the code is arranged in a way that reduces errors due to mutation or mistranslation; that is, amino acids with similar chemical properties are coded by similar codons (Woese 1965; Epstein 1966). Therefore it is possible (Haig and Hurst 1991; Freeland and Hurst 1998; Knight et al. 1999; Freeland et al. 2000) that the main force that shaped the genetic code is selection for minimization of the chemical distances between amino acids, that is, error minimization at the protein level (though this theory is still debated [see Di Giulio 2000a, 2000b; Archetti 2004]).

Since the genetic code is degenerate (there are 64 codons coding for only 20 amino acids and a termination signal), most amino acids are encoded by several synonymous codons, and as Grantham et al. (1980) first demonstrated, and as further data from a number of organisms eventually corroborated, codon usage is not random: some synonymous codons are more used than others. The theory of error minimization for the evolution of the genetic codes postulates that the codons are arranged in the code in a way that reduces errors. The scope of this paper is to test whether error minimization at the protein level plays a role also in the evolution of codon usage in proteincoding genes. The hypothesis is that the preferred codons are the ones that, after mutation or mistranslation, keep on coding for the same amino acid or for amino acids with similar chemical properties.

Following the same logic used to measure the optimization of the genetic code, it is possible to calculate a measure of the capacity to minimize errors due to mutation or mistranslation for each codon. This measure can then be used to calculate the degree 
of error minimization for individual genes or genomes.

If the hypothesis of error minimization for codon usage bias is correct, then not only should the preferred codons be the ones that minimize errors, but also it is expected that there is a correlation between the degree of error minimization and the rate of nonsynonymous nucleotide substitution; that is, highly conserved genes should prefer synonymous codons that minimize the effect of errors. Moreover, the degree of codon usage bias should be correlated with the degree of error minimization, and the amino acids that contribute more to codon usage bias should be the ones whose synonymous codons have a greater variance in the capacity to minimize errors.

I first develop a theoretical, quantitative measure of error minimization for synonymous codons, based on similarities between amino acids; then I use this measure to calculate the degree of error minimization for 82 genes of Drosophila melanogaster and for 432 rodent genes; finally, these values are used to study the relationship of error minimization with the degree of codon usage bias, with the CG content, and with the rates of nucleotide substitution.

\section{Methods}

I developed a computer program that measures the capacity of each codon to minimize the deleterious effects of errors due to mutation or mistranslation. The basic concept is to calculate the mean dissimilarity between the amino acid coded by the original codon and the amino acids coded by its possible mutants; this measure depends only on the structure of the genetic code and on the similarities between amino acids. For the synonymous codons of each amino acid, then, these values of error minimization are correlated with codon usage. The mean value of the correlations is taken as a measure of error minimization for the gene. The method is described in detail in the following sections.

\section{Amino Acid Similarity}

The impact of mutation or mistranslation can be deduced from amino acid similarity matrices. In a similarity scoring matrix, higher values are assigned to more similar pairs of amino acids (George et al. 1990). Throughout this paper I use McLachlan's (1971) classical matrix based on chemical properties, but at the end I compare the results obtained with other matrices.

For each pair of amino acids, I derive the measure $D_{\mathrm{AA} / \mathrm{AA}^{*}}=\omega_{\mathrm{AA} / \mathrm{AA}}-\omega_{\mathrm{AA} / \mathrm{AA}^{*}}$ from the matrix, where $\omega_{\mathrm{AA} / \mathrm{AA}}$ is the similarity of amino acid AA to itself (this value is usually the same for all amino acids, but not in all matrices: in McLachlan's it is either 8 or 9) and $\omega_{\mathrm{AA} / \mathrm{AA}^{*}}$ is the similarity of AA to the mutant amino acid $\mathrm{AA}^{*}$ obtained after an error at one of the three positions of the original codon. Hence, $D_{\mathrm{AA} / \mathrm{AA}^{*}}$ is the distance (dissimilarity) between the original (AA) and the mutant (AA*) amino acid.

Similarities between amino acids and termination signals $\left(\omega_{\mathrm{AA} / \mathrm{STOP}}\right)$ have, of course, no meaning and are not tabulated in similarity scoring matrices; however, a measure of the damage produced by mutations to termination codons must be considered. I use diverse scores for this that are less than or equal to the lowest similarity score of the matrix $(0$ in McLachlan's matrix).

\section{Error Minimization of Synonymous Codons: The MD Value}

Since $\omega_{\mathrm{AA} / \mathrm{AA}} \geq \omega_{\mathrm{AA} / \mathrm{AA}^{*}}$ for every amino acid, $D_{\mathrm{AA} /}$ $\mathrm{AA}^{*}$ is always positive, and the measures of $D_{\mathrm{AA} / \mathrm{AA}^{*}}$ for the possible mutant codons arising by point mutation are positive. Their mean value is taken as a measure of distance (dissimilarity) between the original codon and its possible mutants. I call this measure MD (mean distance). Optimal codons are predicted to have small MD values.

The same procedure can be used to measure the MD between one codon and the possible codons that arise by point mutation after $n$ mutation events. This is important because some synonymous codons have the same MD value after one mutation (Fig. 1). These include most twofold degenerate amino acids and some others such as Pro, because its similarity score with His and Gln is the same (in McLachlan's matrix). In these cases selection for optimal codons will not operate after one mutation but after successive mutation events. Actually, this process can be seen as a differential survival of lineages originating from genomes with different codon usage patterns. For example, consider the part of the progeny that inherited Gly instead of Glu after the first mutation. In the first generation, there is no differential selection between the two lineages with Gly-GGA and Gly-GGG. These two lineages will, however, produce some mutant progeny with the termination signal and Trp, respectively.

I use the following procedure to take into account the importance of each mutation event. I measure MD for each codon and variance of MD for synonymous codons of each amino acid, for every mutation event. For example, the variance after one mutation for Pro is zero, while there is a high variance for the six codons of Leu (Fig. 2). In general, the higher the variance, the higher the intensity of selection. The intensity of selection $(\sigma)$ for each amino acid (AA) for each mutation event $(n)$ is 


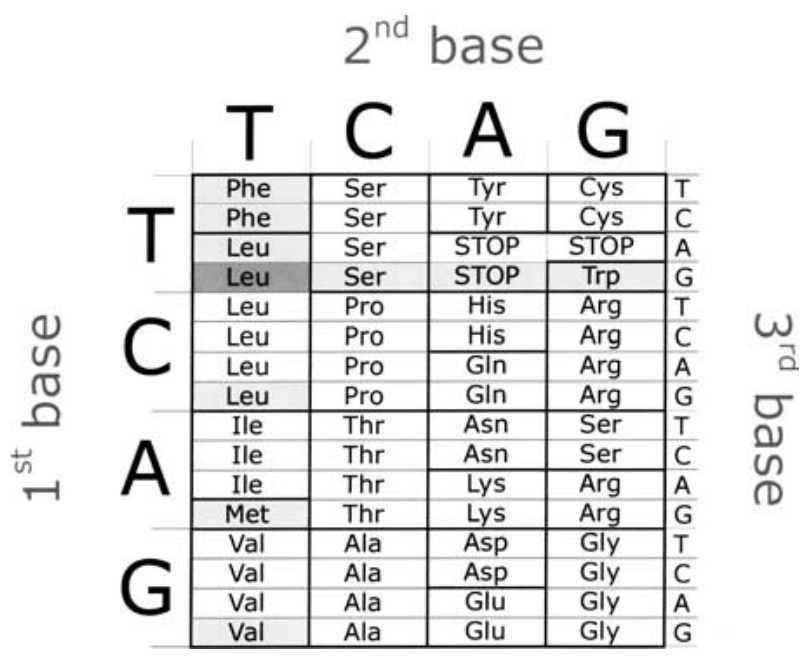

Fig. 1. The standard genetic code. The codons in light gray are the mutant codons originated from the original codon (in dark gray) after one point mutation.

$$
\sigma_{\mathrm{AA}(n)}=\frac{v_{\mathrm{AA}(n)}}{\sum_{m=1}^{10} v_{\mathrm{AA}(m)}}
$$

where $v_{\mathrm{AA}(n)}$ is the variance of $\mathrm{MD}$ values for synonymous codons coding for amino acid AA after the $n$th mutation. I consider mutations up to the 10th: although this is an arbitrary limit, MD values clearly converge to the same value, for all codons, as $n$ grows (Fig. 2) because there are $9^{n}$ possible mutants but only 64 codons; therefore after many mutation events the differences of MD values for different codons become negligible.

The importance of mutations also depends on the order. Further mutations are likely to be less important if selection had already occurred in previous generations. I use the following simple method (a decision on how to weight successive mutations is likely to be arbitrary; however, different methods gave very similar results): for each amino acid (AA), the weight $(\Omega)$ of the first mutation is equal to its intensity of selection $\left[\Omega_{\mathrm{AA}(1)}=\sigma_{\mathrm{AA}(1)}\right]$, while for the successive mutations, at the $n$th mutation, the weight is

$$
\Omega_{\mathrm{AA}(n)}=\left[1-\sum_{2}^{n} \sigma_{\mathrm{AA}(n-1)}\right] \sigma_{\mathrm{AA}(n)}
$$

Imagine, for example, that $\sigma_{\mathrm{AA}(1)}=0.2, \sigma_{\mathrm{AA}(2)}=0.7$, $\sigma_{\mathrm{AA}(3)}=0.1$, and $\sigma_{\mathrm{AA}(\mathrm{n})}=0$ for all $n>3$. The corresponding weights will be $\Omega_{\mathrm{AA}(1)}=0.2$, $\Omega_{\mathrm{AA}(2)}=0.56$, and $\Omega_{\mathrm{AA}(3)}=0.01$. The corresponding MD values are multiplied by these weights and then summed (up to the 10th mutation, unless otherwise stated in the text).

\section{Mutation Bias}

The values taken from the matrix can be weighted also by the probability that such mutations occur.

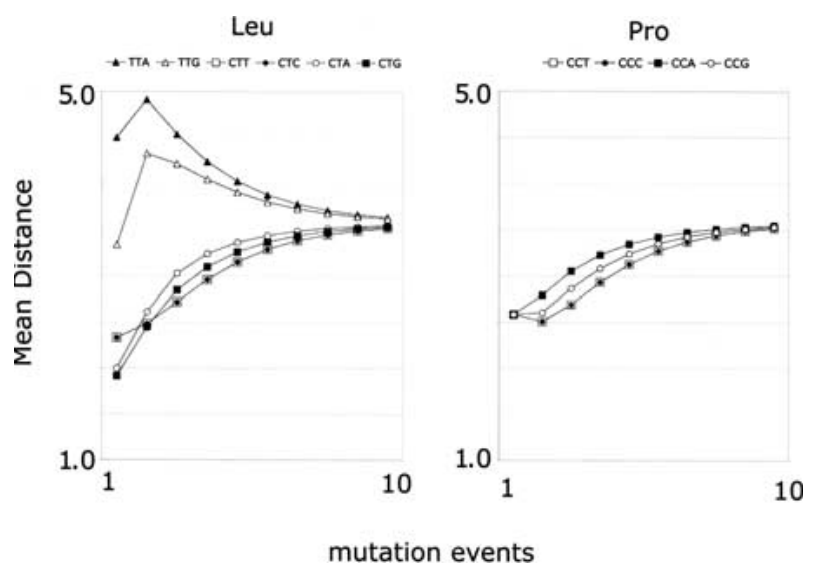

Fig. 2. MD values for the codons of Leu and Pro after further mutation events (no transition/transversion bias; CG/AT mutation ratio $\left.=1 ; \omega_{\mathrm{AA} / \mathrm{STOP}}=-10\right)$.

Transitions $\quad(\mathrm{C} \leftrightarrow \mathrm{T}, \quad \mathrm{A} \leftrightarrow \mathrm{G})$ and transversions $(\mathrm{C}, \mathrm{T} \leftrightarrow \mathrm{A}, \mathrm{G})$ are not equally likely to occur (Topal and Fresco 1976). Moreover, it is also possible that accuracy varies during translation according to base position within a codon (Woese 1965), since the translation machinery acts upon mRNA in a specific reading frame, reading bases in triplets. Therefore, before calculating $\mathrm{MD}, D_{\mathrm{AA} / \mathrm{AA}} *$ can be multiplied by the probability of a change from the original base to the mutant one (allowing for a consideration of whether it is a transition or a transversion) and by the probability of mistranslation based on its position on the codon (1st, 2 nd, or $3 \mathrm{rd}$ ).

I have followed the same assumptions used by Freeland and Hurst (1998) to test the optimization of the genetic code, namely, that mistranslation of the second position is much less frequent than mistranslation of the first or third position; that mistranslation of the first position is less frequent than mistranslation of the third codon position; that mistranslations at the second position are almost exclusively transitional; that mistranslation at the first position is biased towards transitional errors; and that there is very little transition bias at the third position. The precise values used to weight each mutation are summarized in Table 1.

As with translation, different rates for transition and transversion must be considered for mutation but in this case there is no reason to assume that these rates vary depending upon base position (see Table 1).

Moreover, I consider the possibility of different mutation rates for $\mathrm{CG}$ and $\mathrm{AT}$, because $\mathrm{C}$ and $\mathrm{G}$, which have three hydrogen bonds, may be more stable than A or T.

\section{Error Minimization for Individual Genes}

Since MD is a measure of dissimilarity, lower values of MD correspond to optimal codons (codons that 
Table 1. Relative frequencies of mutation and mistranslation used in the model

\begin{tabular}{|c|c|c|c|c|c|c|c|}
\hline & \multicolumn{6}{|c|}{ Mistranslation } & \multirow{3}{*}{$\begin{array}{l}\text { Mutation } \\
\text { (all bases) } \mathrm{T} / \mathrm{T} \text { ratio }\end{array}$} \\
\hline & \multicolumn{3}{|c|}{ Frequency $^{\mathrm{a}}$} & \multicolumn{3}{|c|}{$\mathrm{T} / \mathrm{T}$ ratio $^{\mathrm{b}}$} & \\
\hline & $1 \mathrm{st}$ & $2 \mathrm{nd}$ & $3 \mathrm{rd}$ & $1 \mathrm{st}$ & $2 \mathrm{nd}$ & $3 \mathrm{rd}$ & \\
\hline A & 0.5 & 0.1 & 1 & 2 & 5 & 1 & - \\
\hline B & - & - & - & - & - & - & 1.5 \\
\hline $\mathrm{C}$ & 0.5 & 0.1 & 1 & 2 & 5 & 1 & 1.5 \\
\hline
\end{tabular}

${ }^{\mathrm{a}}$ Frequency of mistranslation for the first, second, or third base of the codon.

${ }^{\mathrm{b}}$ Transition/transversion ratio (relative to a transversion rate $=1$ ) for the first, second, or third base of the codon.

minimize the effects of errors). MD measures can be compared only within each synonymous family (amino acid) and not between different amino acids, because each position in the sequence of a protein must be occupied by a certain amino acid, and codons for that amino acid can be chosen only among synonymous codons. The sample size is too small $(n=2$ to 6$)$ to yield a significant correlation within each amino acid, but the importance of error minimization can be measured by the sum of the correlations between $\mathrm{MD}$ and synonymous codon frequencies for all the 18 degenerate amino acids. This measure $(R)$ ranges between $-N$ and $+N$, where $N$ is the number of amino acids on which the correlations are measured (for the standard genetic code, usually $N=18$, but $N<18$ if for some amino acid there is no variance in the MD values or in the frequency of its synonymous codons). A normalized version of $R\left(R_{N}\right.$ : from -1 to +1$)$ can be obtained by dividing $R$ by $N$; both measures can be transformed weighting the correlation of each amino acid by its frequency $\left(w R\right.$ and $\left.w R_{N}\right) . R$ and $w R$ values close to $-N$ (or $R_{N}$ and $w R_{N}$ values close to -1 ) will mean a strong tendency to minimize the effects of errors.

\section{Genes and Other Measures Used in the Analysis}

Codon usage and the degree of codon usage bias for individual genes are calculated from the coding sequences of 82 D. melanogaster genes and 432 mouserat homologs. Codon usage for the entire genome of D. melanogaster is taken from the Codon Usage Database (www://kazusa.or.jp/codon; described by Nakamura et al. 2000). The measure used for the degree of codon usage bias is the effective number of codons (ENC; Wright 1990); ENC values range from 20 (high bias - only one codon per amino acid is used) to 61 (low bias - all codons are used equally).

For Drosophila, the genes are the same used by Moriyama and Powell (1997), while the substitution rates are taken from Akashi (1994) for a limited set of genes. Genes and substitution rates for rodents are the ones used in Smith and Hurst (1997). $K_{a}$ and $K_{s}$ are the rates of synonymous and nonsynonymous substitution, $K_{4}$ is the rate of nonsynonymous substitution at fourfold sites $\left(K_{4}\right.$ is more reliable than $K_{s}$ because it does not have to combine rates of sites with different degeneracies).

\section{Random Sequences}

To study the importance of CG content, I also use random sequences (82 for Drosophila, 432 for rodents) with the same length and the same CG content as the real genes (for rodents I use the CG content of the mouse genes). I also use random sequences with the same CG content (as the real genes) at each of the three positions. Both these kinds of sequences maintain the same CG content, but not the same degree of codon usage bias (ENC).

As a null model in the study of the degree of codon usage bias I use, instead, sequences (82 for Drosophila, 432 for rodents) with the same length and the same ENC values as the real genes, produced by switching at random the frequencies of synonymous codons. These sequences maintain the same ENC of the real genes but not the same CG content.

\section{Results}

\section{Values}

Table 2 shows MD values obtained with different values of the parameters. With no transition/transversion bias and with the same mutation rate for CG and AT, some amino acids show an ambiguity about which codons have the lowest MD value. This reflects the arrangement of the codons in the code (Fig. 1): in the standard code with no bias, for example, all twofold degenerate amino acids except Glu, Lys, and Gln have no variance in MD values for their synonymous codons because each couple of codons can mutate only to new couples of codons that are synonymous as well (and if only the first mutation event is considered, only codons for Lys have different MD values because one of them can mutate to Met and the other one to Ile-but, incidentally, in McLach- 
404

Table 2. MD values

\begin{tabular}{|c|c|c|c|c|c|c|}
\hline \multicolumn{2}{|c|}{ No bias, ${ }^{\text {a }} 1$ mutation only } & \multicolumn{2}{|c|}{ No bias } & \multirow{2}{*}{$\begin{array}{l}\mathrm{A}^{\mathrm{a}} \\
(\chi=0.9)\end{array}$} & \multirow{2}{*}{$\begin{array}{l}\mathrm{B}^{\mathrm{a}} \\
(\chi=0.9)\end{array}$} & \multirow{2}{*}{$\begin{array}{l}\mathrm{C}^{\mathrm{a}} \\
(\chi=0.9)\end{array}$} \\
\hline$\chi^{\mathrm{b}}=0.9$ & $\chi=1$ & $\chi=0.9$ & $\chi=1$ & & & \\
\hline
\end{tabular}

$\operatorname{Arg}$



No bias, ${ }^{\text {a } 1 \text { mutation only } \quad \text { No bias }}$

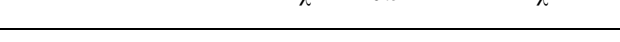

Leu

Gly

$\begin{array}{ll}\text { CGA } & 2.775 \\ \text { CGC } & 2.250 \\ \text { CGG } & 1.800 \\ \text { CGT } & 2.250 \\ \overline{\mathrm{AGA}} & \underline{3.708} \\ \overline{\mathrm{AGG}} & 2.558\end{array}$

\begin{tabular}{ll}
3.083 & 1.947 \\
2.500 & 1.552 \\
2.000 & 1.411 \\
2.500 & 1.601 \\
$\underline{3.833}$ & $\underline{2.422}$ \\
\hline 2.750 & 1.805
\end{tabular}

\subsection{1}

\subsection{7}

$1.898 \quad 1.002$

1.662

0.754

1.898

1.009

$\frac{2.781}{2.099}$

$\frac{2.076}{1.258}$

1.696

2.000

1.567

$\begin{array}{ll}\text { CTA } & 1.950\end{array}$

2.333

1.524

CTG $\quad \mathbf{1 . 8 7 5}$

CTT 2.258

$\begin{array}{ll}\text { TTA } & \frac{4.500}{3.283}\end{array}$

1.917
2.333

1.438

1.577

$\frac{4.500}{3.333} \quad \frac{3.023}{2.445}$

1.719

1.609

1.719

$\frac{3.172}{2.652}$

2.799

TCA $\quad 4.150$

4.500

2.417

$\begin{array}{ll}\text { TCC } & 2.275 \\ \text { TCG } & 3.175\end{array}$

$\begin{array}{ll}\text { TCT } & 2.275\end{array}$

$\overline{\text { AGC }} \quad 2.750$

AGT 2.817

3.417

1.745

2.243

2.417

2.917

1.798

1.881

2.917

1.957

3.172

1.989

2.578

1.989

2.119

2.119

\subsection{4}

0.762

0.631

0.770

$\frac{1.745}{1.315}$

$\frac{2.333}{2.167}$

$\frac{1.705}{1.457}$

$\frac{1.998}{1.785}$

1.535

2.333

1.566

1.911

1.785

1.816

1.045

1.414

1.048

1.660

1.717

1.558

2.583

$\mathbf{1 . 3 3 7}$

$\begin{array}{ll}\mathrm{CCC} & 2.325\end{array}$

2.583

2.583

$\begin{array}{ll}\mathrm{CCG} & 2.325 \\ \mathrm{CCT} & 2.325\end{array}$

1.391

1.444

$\frac{0.717}{\mathbf{0 . 6 0 7}}$

0.651

0.635

2.071

0.518

1.866

$\mathbf{0 . 4 5 1}$

$1.958 \quad 0.465$

2.583

1.866

0.481

$\frac{2.035}{1.838}$

$\underline{0.631}$

2.333

$\frac{1.552}{1.335}$

2.250

1.391

GCG 2.025

GCT 2.100

1.439

1.934

1.838

0.563

0.559

2.637

1.737

2.153

GGC $\quad \mathbf{2 . 4 7 5}$

3.667

2.750

1.654

GGG 2.475

GGT 2.475

2.750

1.677

2.043

1.137

2.068

1.134

1.145

2.167

$\begin{array}{ll}\text { GTA } & 2.092 \\ \text { GTC } & 2.325 \\ \text { GTG } & 2.167\end{array}$

2.417

2.250

GTT 2.325

2.417

$\frac{1.686}{1.493}$

1.526

1.607

$\begin{array}{ll}\frac{1.928}{1.856} & 0.756 \\ 1.878 & 0.849\end{array}$

1.878

0.761

1.856

0.856

$\frac{2.167}{1.990}$

0.809

$\frac{1.941}{\mathbf{1 . 6 7 4}}$

$\mathbf{0 . 7 8 3}$

1.990

0.816

$\frac{2.671}{2.626}$

$\frac{1.247}{1.176}$

2.250

1.009

1.841

2.250

1.082

$\frac{2.632}{2.562}$

$\frac{1.003}{0.947}$

2.203

0.826

2.203

0.880

$\begin{array}{r}1.650 \\ \hline\end{array}$

2.833

$\frac{1.992}{\mathbf{1 . 8 0 0}}$

7.356

6.477

6.450

7.105

$\frac{8.520}{7.423}$

7.423

5.938

5.319

5.326

5.789

$\frac{8.271}{7.177}$

7.177

7.929

6.565

6.899

7.173

$\mathbf{6 . 4 2 4}$

7.019

2.814

2.429

2.339

2.584

$\frac{3.603}{2.885}$

2.522

2.343

2.291

2.459

5.124

4.464

2.744

3.491

2.889

2.681

2.823

$\frac{10.290}{\mathbf{9 . 1 5 4}}$

9.209

10.107

$\frac{3.565}{\mathbf{3 . 0 4 4}}$

3.158

3.314

9.459

3.306

$\mathbf{8 . 4 3 2}$

$\mathbf{2 . 8 8 7}$

8.469

9.316

2.941

9.339

$\mathbf{8 . 3 1 2}$

8.357

9.178

7.989

6.976

6.993

7.659

3.162

$\underline{3.287}$

2.829

2.911

3.084

3.226

2.610

2.611

2.775

$\frac{10.467}{9.329}$

$\underline{3.684}$

9.370

10.302

3.272

3.502

$\frac{11.176}{9.941}$

$\frac{4.041}{3.465}$

10.972

3.773

11.913

$\underline{4.478}$

3.999

11.314

4.045

$\underline{12.510}$

4.451

$\frac{10.693}{9.585}$

$\frac{3.906}{\mathbf{3 . 4 9 7}}$

CAT 2.733 
Table 2. Continued

\begin{tabular}{|c|c|c|c|c|c|c|}
\hline \multicolumn{2}{|c|}{ No bias, ${ }^{\text {a }} 1$ mutation only } & \multicolumn{2}{|c|}{ No bias } & \multirow[b]{2}{*}{$\begin{array}{l}\mathrm{A}^{\mathrm{a}} \\
(\chi=0.9)\end{array}$} & \multirow{2}{*}{$\begin{array}{l}\mathrm{B}^{\mathrm{a}} \\
(\chi=0.9)\end{array}$} & \multirow[b]{2}{*}{$\begin{array}{l}\mathrm{C}^{\mathrm{a}} \\
(\chi=0.9)\end{array}$} \\
\hline$\chi^{\mathrm{b}}=0.9$ & $\chi=1$ & $\chi=0.9$ & $\chi=1$ & & & \\
\hline
\end{tabular}

\begin{tabular}{|c|c|c|c|c|c|c|c|c|}
\hline \multicolumn{9}{|l|}{ Glu } \\
\hline & GAA & 3.625 & 3.833 & 2.001 & 2.639 & 1.082 & 10.715 & 4.025 \\
\hline & $\overline{\text { GAG }}$ & 3.575 & 3.833 & 1.807 & 2.584 & 1.016 & 9.602 & 3.595 \\
\hline \multicolumn{9}{|l|}{ Asp } \\
\hline & GAC & 2.917 & 3.083 & 1.666 & 2.244 & 0.884 & 10.196 & 3.650 \\
\hline & $\overline{\mathrm{GAT}}$ & 2.967 & 3.083 & 1.816 & 2.244 & 0.948 & 11.273 & 4.015 \\
\hline \multicolumn{9}{|l|}{ Tyr } \\
\hline & TAC & 5.933 & 6.250 & 2.755 & 3.087 & 2.885 & 11.133 & 4.440 \\
\hline & $\underline{\text { TAT }}$ & $\underline{6.250}$ & 6.250 & $\underline{2.975}$ & 3.087 & $\underline{3.091}$ & 12.295 & $\underline{4.860}$ \\
\hline \multicolumn{9}{|l|}{ Cys } \\
\hline & TGC & 5.667 & 6.083 & 2.366 & 2.969 & 2.320 & 10.066 & 3.935 \\
\hline & TGT & 5.883 & 6.083 & 2.552 & 2.969 & 2.464 & 11.119 & 4.310 \\
\hline \multicolumn{9}{|l|}{ Phe } \\
\hline & TTC & 3.517 & 3.583 & 1.956 & 2.415 & 0.968 & 11.368 & 4.290 \\
\hline & $\underline{\text { TTT }}$ & $\underline{3.583}$ & 3.583 & $\underline{2.130}$ & 2.415 & $\underline{1.027}$ & $\underline{12.567}$ & $\underline{4.715}$ \\
\hline
\end{tabular}

Note. The most used codons for Drosophila melanogaster are in boldface; the less used codons are underlined. MD values are typed in boldface/underlined when they correspond to the most/less used codon and in italics when the case is ambiguous. $\omega_{\mathrm{AA} / \mathrm{STOP}}=-10$.

${ }^{\mathrm{a}} \mathrm{A}$, mistranslation only; B, mutation only; $\mathrm{C}$, mutation and mistraslation (parameters as in Table 1).

${ }^{\mathrm{b}} \chi=\mathrm{CG} / \mathrm{AT}$ mutation ratio.

lan's matrix $\omega_{\text {Lys/Ile }}=\omega_{\text {Lys/Met }}$, so even Lys has no variance for $\mathrm{MD}$ after one mutation). Introducing a bias in the mutation rate for CG versus AT, or in the transition/transversion ratio, produces a higher variability in the MD values.

The measures of MD also depend on the "similarity" with the termination signal $\left(\omega_{\mathrm{AA} / \mathrm{STOP}}\right)$. The relative differences of MD values for synonymous codons depend slightly on $\omega_{\mathrm{AA} / \mathrm{sTOP}}$ (Fig. 3). Therefore, in the analysis I use $\omega_{\mathrm{AA} / \mathrm{STOP}}$ values ranging from 0 to -50 ( 0 is the lowest value in McLachlan's matrix, in which scores range from 0 to 9 ).

\section{Error Minimization}

It can already be noted (Table 2, Fig. 4) that in the genome of $D$. melanogster, in most cases the optimal codons (the ones with the lowest MD values) correspond to the most used codons and the worst codons (the ones with the highest MD values) correspond to the less used codons. The main exception is apparently Ser, for which AGC is the most frequent codon, but the MD value for AGC is not the lowest (it is the lowest, however, if the transition/transversion bias is considered). Also, the preferred codons for Arg and Val do not correspond to the lowest MD values, the less used codons in Gly and Pro do not correspond to the highest MD values, and the values are inverted in Asp.

Codon usage frequencies (obtained from the Codon Usage Database) are quite rough measures of codon preference in the whole genome. Moriyama and Powell (1997) use, as a measure of codon preference in Drosophila, the correlation between the degree of bias for more than 1000 genes and the frequency of T-, C-, A-, or G-ending codons within each gene (positive values indicate codons that are increasingly used as the codon usage bias for that amino acid increase). Moreover, to avoid stochastic fluctuations, Moriyama and Powell (1997) examine only genes longer than $200 \mathrm{bp}$. These values should therefore give more reliable information about the preference of synonymous codons than mere usage percentages. When these measures are used there is a difference with Ser: in this case the preferred codon is TCC, which indeed corresponds to the lowest MD value with no transition/transversion bias, while the lowest value in Moriyama and Powell is for TCA, which corresponds to the highest MD value. There are also minor differences between codon frequency (from the Codon Usage Database) and Moriyama and Powell measures for Arg and Gly.

The pattern for rodents is quite similar. For a quantitative measure of error minimization from now on I use mainly the $w R_{N}$ values (described in the Methods section), but results obtained with $R_{N}$ are quite similar. If error minimization based on the similarity between amino acids plays no role in the evolution of the genes analyzed, then $w R_{N}$ (or $R_{N}$ ) values should have a Gaussian distribution centered in zero. A deviation from this distribution will indicate a tendency toward error minimization (if $w R_{N}$ values are more frequent toward -1 ) or maximization (toward +1 ).

Figure 5 shows that there is a strong prevalence of low $w R_{N}$ values, that is, the genes analyzed prefer codons that reduce the impact of errors at the protein level, both for Drosophila and for rodents. A very 
Leu

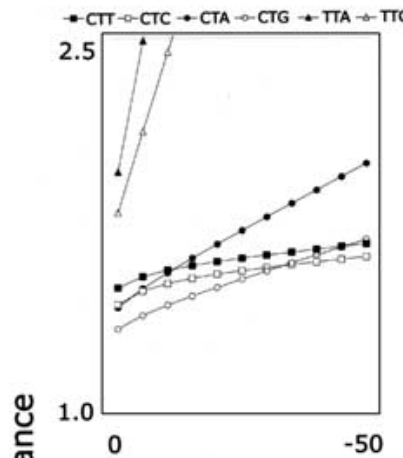

Gly

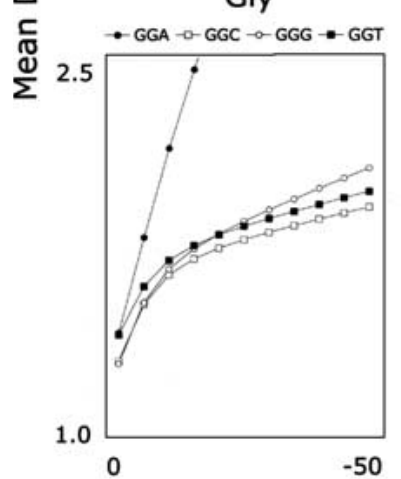

Ser

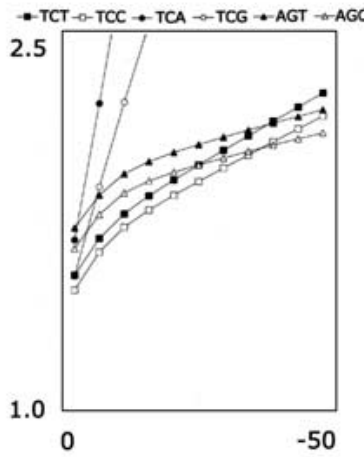

Val

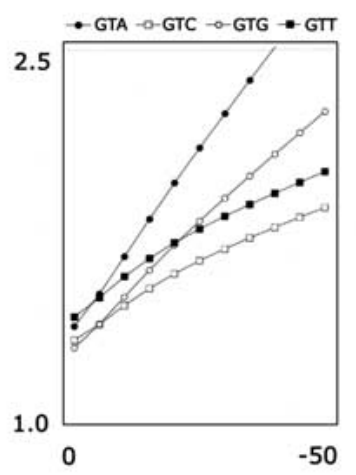

WAA/STOP

Fig. 3. MD values for the codons of Leu, Ser, Gly, and Val, for different values of $\omega_{\mathrm{AA} / \mathrm{STOP}}$ (no transition/transversion bias; $\mathrm{CG} /$ $\mathrm{AT}$ mutation ratio $=0.9$ ).

similar pattern is obtained with $R_{N}$ values (not shown; see Appendix for Drosophila $w R_{N}$ values; values for rodents are available on request). Note that even if the frequency of very low $w R_{N}$ values (close to -1 ) is not high, this does not necessarily mean that error minimization is weak; the comparison must be done with the expected random distribution or, better, with the distribution obtained with random sequences with the same CG content (see next section).

These results do not change much when a bias in the transition/transversion rate is considered for mutation, mistranslation, or both or different values of the similarity score with the termination signal $\left(\omega_{\mathrm{AA} / \mathrm{STOP}}\right)$, ranging between 0 and -50 , are used. The CG/AT mutation ratio also does not seem to have a strong influence, especially for rodents (Fig. 6). Finally, when only one mutation event is considered, the distribution of $w R_{N}$ values is less biased toward error minimization (Fig. 6). This might mean that multiple mutation events, that is, selection on mutations rather than on mistranslation, are important in error minimization at the protein level.

In general, it seems that both Drosophila and rodent genes prefer codons that reduce the impact of errors.

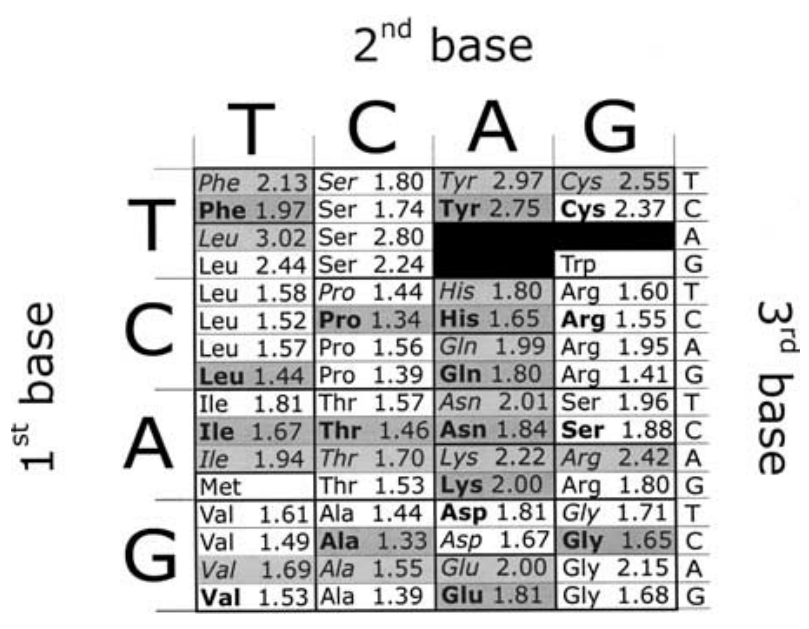

Fig. 4. The standard genetic code with $\mathrm{MD}$ values $\left(\omega_{\mathrm{AA} / \mathrm{STOP}}\right.$ $=-10 ; \mathrm{CG} / \mathrm{AT}$ mutation ratio $=0.9$; no transition/transversion bias). Values at the right in each cell are the MD values for the corresponding codon. Codons in boldface/italics are the most/less used in Drosophila melanogaster. Cases where the lowest/higest MD values correspond to the most/less used codons are marked in dark/light gray.

\section{Error Minimization and CG Content}

It is possible, in principle, that $w R_{N}$ (or $R_{N}$ ) values are correlated with $C G$ content, and a bias in their values might simply be the by-product of a mutational bias. To investigate this possibility, I produce random sequences (82 for Drosophila,432 for rodents), 300 codons long, with exactly the same CG content as the real genes analyzed here (for rodents I use the CG content of the mouse genes, but results are virtually identical for rat genes). If CG content, irrespective of the specific codons used, was the main cause of the pattern observed, then the distribution of $w R_{N}$ (or $R_{N}$ ) values for these random sequences would be similar to the distribution of $w R_{N}$ (or $R_{N}$ ) values for the real genes.

Figure 5 shows that $w R_{N}$ values for sequences with "random" codons are almost Gaussian, not biased and very different from the $w R_{N}$ values of the real genes. A similar result is obtained when using position-specific $\mathrm{CG}$ content.

Moreover, CG content is not correlated with $w R_{N}$ in the real genes (see Table 3). These results do not change much with a bias in the transition/transversion or CG/AT mutation rate or with different values of the similarity score with the termination signal $\left(\omega_{\mathrm{AA} / \mathrm{STOP}}\right)$, ranging between 0 and -50 (Table 3$)$.

Since $w R_{N}$ values are obtained weighting the frequencies of amino acids, this may lead to an underestimation of the importance of some amino acids and an overestimation of some others; it may be thought, therefore, that these results depend on amino acid frequencies, and that the random sequences fail to show error minimization because they have a deficit in some amino acid. However, though I have discussed mainly results obtained with $w R_{N}$ 


\section{D. melanogaster}

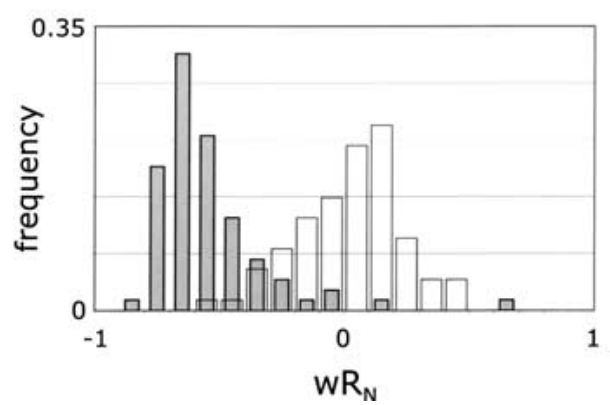

M. musculus

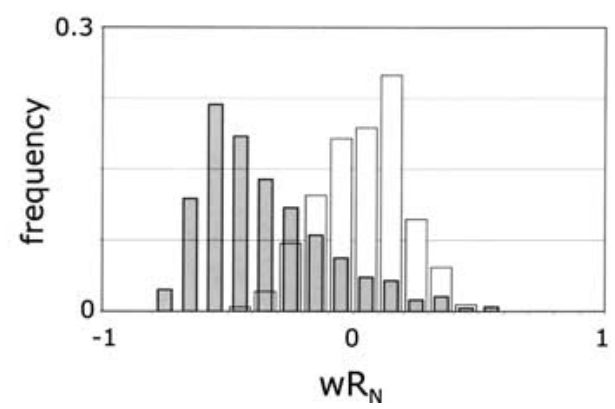

Fig. 5. Distribution of the $w R_{N}$ values for the real genes (gray) and for random sequences with the same CG content (white).

\section{D. melanogaster}
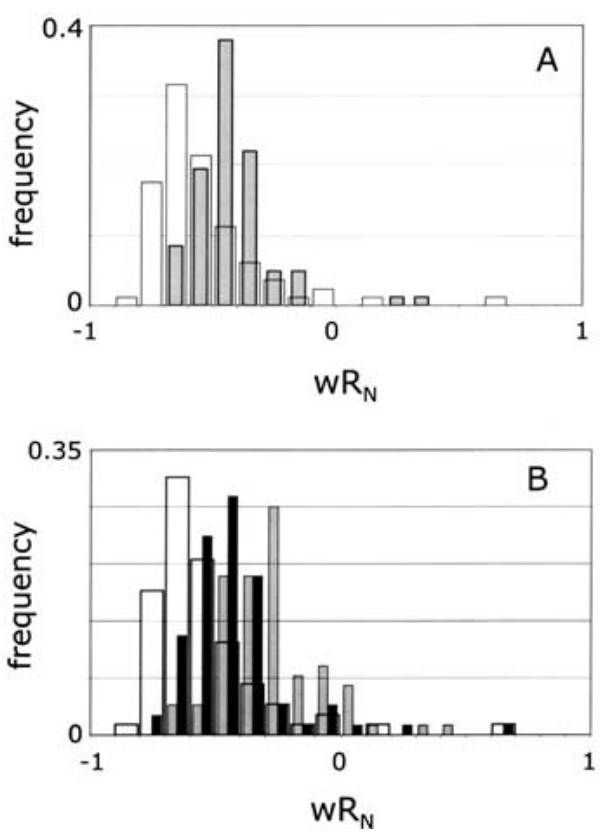

M. musculus
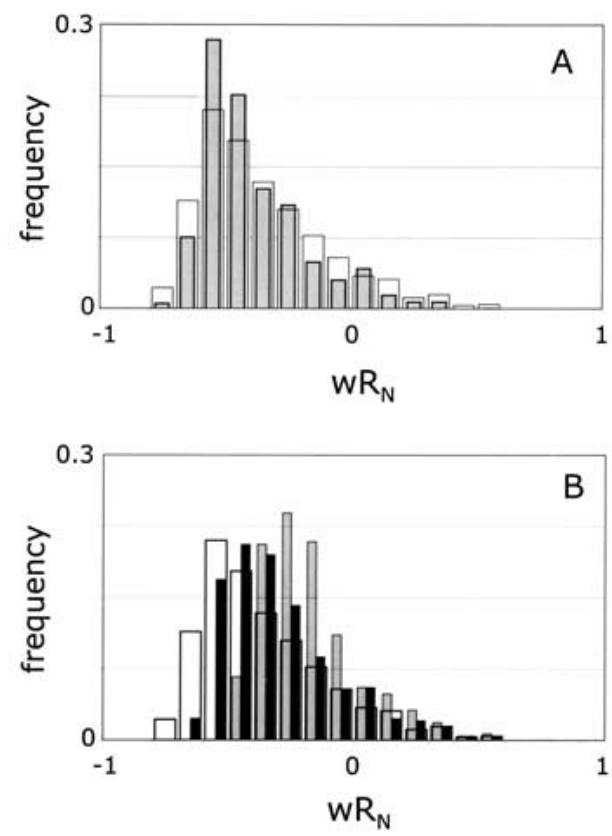

Fig. 6. Distribution of the $w R_{N}$ values for the real genes obtained with different CG/AT mutation ratios $(\mathbf{A}$; white $=0.9$, gray $=1)$ or with a different number of mutation events $(\mathbf{B}$; white $=10$, black $=2$, gray $=1)\left(\omega_{\mathrm{AA} / \mathrm{STOP}}=-10\right.$; no transition $/$ transversion bias; CG $/ \mathrm{AT}$ mutation ratio $=0.9)$.

values, the results obtained with $R_{N}$ values are very similar. $R_{N}$ values do not depend on the frequency of amino acids, as all amino acids are weighted the same. The use of $R_{N}$ may also be questionable, however, because the more frequent an amino acid, the more likely it is to influence selection on that protein. In any case, the results obtained using $w R_{N}$ and $R_{N}$ are very similar.

Therefore, it seems that it is the specific choice of codons, and not solely $\mathrm{CG}$ content on amino acid composition, that determines error minimization. That is, error minimization is not due to mutation bias.

\section{Error Minimization and Codon Usage Bias}

If codon usage bias is influenced by the degree of error minimization, then it should be possible to find a correlation between the variance of MD values for synonymous codons and the contribution of each individual amino acid to the overall codon usage bias. Moreover, it should be possible to find a correlation between the degree of error minimization and the degree of codon usage bias for individual genes. I analyze these two aspects in turn.

Variance of MD and Contribution to the Bias. Moriyama and Powell (1997) report the contribution to the bias of each amino acid for Drosophila. A correlation between the variance of MD values for synonymous codons (the measure developed here) and the contribution of the corresponding amino acid to codon usage bias (taken from Moriyama and Powell 1997) is expected because natural selection for error minimization, if it actually 
Table 3a. Correlation between $w R_{N}$ and CG content, ENC, $K_{a}$, and $K_{s}$ for Drosophila

\begin{tabular}{|c|c|c|c|c|}
\hline \multirow[b]{2}{*}{ Parameters $^{\mathrm{b}}$} & \multicolumn{4}{|c|}{ Correlation of $\mathrm{w} R_{N}$ with $^{\mathrm{a}}$} \\
\hline & $\% \mathrm{CG}$ & ENC & $K_{s}$ & $K_{a}$ \\
\hline$\omega_{\mathrm{AA} / \mathrm{STOP}}=0$ & -0.02 & $0.51^{* * * * *}$ & $0.44 * *$ & $0.54 * * *$ \\
\hline$\omega_{\mathrm{AA} / \mathrm{STOP}}=-10$ & -0.11 & $0.60 * * * *$ & $0.56 * * *$ & $0.43^{*}$ \\
\hline$\omega_{\mathrm{AA} / \mathrm{STOP}}=-20$ & -0.18 & $0.61^{* * * * *}$ & $0.58 * * * * *$ & $0.38 *$ \\
\hline$\omega_{\mathrm{AA} / \mathrm{STOP}}=-50$ & -0.21 & $0.60 * * * *$ & $0.57 * * * *$ & $0.33^{*}$ \\
\hline $\operatorname{Bias}=A^{c}$ & -0.12 & $0.56 * * * *$ & $0.51 * * *$ & $0.35^{*}$ \\
\hline Bias $=B^{c}$ & -0.02 & $0.51 * * * *$ & $0.54 * * *$ & $0.45^{* *}$ \\
\hline Bias $=C^{c}$ & -0.06 & $0.56 * * * *$ & $0.50^{* * *}$ & $0.47 * *$ \\
\hline$\chi=1$ & -0.21 & $0.48 * * * *$ & $0.55 * * *$ & $0.44 * *$ \\
\hline 1 mutation & 0.21 & $0.32 *$ & 0.24 & $0.44 * *$ \\
\hline 2 mutations & -0.14 & $0.51 * * * *$ & $0.53^{* * *}$ & $0.40^{*}$ \\
\hline
\end{tabular}

Table 3b. Correlation between $w R_{N}$ and $\mathrm{CG}$ content, ENC, $K_{a}$, and $K_{s}$, for the 432 rodent genes

Correlation of $w R_{N}$ with $^{\mathrm{a}}$

\begin{tabular}{|c|c|c|c|c|}
\hline \multirow[b]{2}{*}{ Parameters $^{\mathrm{b}}$} & \\
\hline & $\% \mathrm{CG}$ & $\mathrm{ENC}$ & $K_{s}$ & $K_{a}$ \\
\hline$\omega_{\mathrm{AA} / \mathrm{STOP}}=0$ & 0.04 & $0.33 * * * *$ & -0.02 & $0.19 * * * *$ \\
\hline$\omega_{\mathrm{AA} / \mathrm{STOP}}=-10$ & -0.03 & $0.32 * * * *$ & -0.01 & $0.20 * * * *$ \\
\hline$\omega_{\mathrm{AA} / \mathrm{STOP}}=-20$ & -0.06 & $0.29 * * * *$ & -0.01 & $0.20 * * * *$ \\
\hline$\omega_{\mathrm{AA} / \mathrm{STOP}}=-50$ & -0.08 & $0.28 * * * *$ & 0.00 & $0.20 * * * *$ \\
\hline $\operatorname{Bias}=\mathrm{A}^{\mathrm{c}}$ & -0.04 & $0.34 * * * *$ & 0.00 & $0.21 * * * *$ \\
\hline Bias $=\mathrm{B}^{\mathrm{c}}$ & 0.04 & $0.34 * * * *$ & -0.01 & $0.19 * * * *$ \\
\hline Bias $=\mathrm{C}^{\mathrm{c}}$ & -0.03 & $0.32 * * * *$ & -0.01 & $0.20 * * * *$ \\
\hline$\chi=1$ & $-0.18 * * *$ & $0.24 * * * *$ & -0.00 & $0.23 * * * *$ \\
\hline 1 mutation & 0.04 & $0.31 * * * *$ & 0.01 & $0.10 *$ \\
\hline 2 mutations & -0.05 & $0.32 * * * *$ & 0.01 & $0.21 * * * *$ \\
\hline
\end{tabular}

Note. The correlation with $\% \mathrm{CG}$ and with ENC for Drosophila is calculated for all 82 genes; the correlation with $K_{s}$, and $K_{a}$, only for a subset of 38 genes (see Appendix). Significant correlations are in boldface $(* p<0.05 ; * * p<0.005 ; * * * p<0.001 ; * * * *<0.0001)$.

${ }^{\mathrm{a}} \% \mathrm{CG}, \mathrm{CG}$ content; ENC, effective number of codons; $K_{s}$ and $K_{a}$, rate of synonymous and nonsynonymous substitution.

${ }^{\mathrm{b}}$ Unless otherwise stated, $\omega_{\mathrm{AA} / \mathrm{STOP}}=-10 ; \chi(=\mathrm{CG} / \mathrm{AT}$ mutation ratio $)=0.9$; no bias in the transition/transversion ratio; $\mathrm{MD}$ values calculated up to the 10 th mutation event.

${ }^{\mathrm{c}}$ Parameters as in Table 1.

plays a role in the evolution of coding sequences, will be stronger for amino acids whose codons have a high variance in the capacity to minimize errors.

Table 4 shows that there is a positive correlation between the variance of MD values and the contribution of each amino acid to the overall codon usage bias. There is a weak, but significant, correlation if one considers all the 18 degenerate amino acids; the correlation is stronger if one does not consider twofold degenerate amino acids. The transition/transversion ratio and the $\omega_{\mathrm{AA} / \mathrm{STOP}}$ value do not seem to affect the results significantly. A higher mutation rate for AT versus CG is necessary for a significant correlation if one considers all 18 degenerate amino acids. The reason why the correlation is more significant when we do not consider twofold degenerate amino acids is probably because variance for twofold degenerate amino acids is usually very low or zero.

Degree of Error Minimization and Degree of Codon Usage Bias. If codon usage is influenced by selection for error minimization at the protein level, then a positive correlation is expected between the degree of codon usage bias and the degree of error minimization. That is, genes with a highly biased codon usage should be those with a strong preference for codons that minimize the effect of errors.

For "random" sequences with the same CG content as the 82 Drosophila genes, the degree of error minimization is not correlated with the degree of codon usage bias (ENC values) $(r=-0.07$, $p=0.70$, for $w R_{N}$ values; $r=0.09, p=0.62$, for $R_{N}$ values) ( $\omega_{\mathrm{AA} / \mathrm{STOP}}=-10 ; \mathrm{CG} / \mathrm{AT}$ mutation bias $=0.9$; no transition/transversion bias). On the other hand, there is a positive correlation (Fig. 7, Table $3 \mathrm{a}$ ) between $w R_{N}$ (or $R_{N}$ ) values and the degree of codon usage bias (ENC) for the real 82 Drosophila genes considered.

A correlation between the degree of codon usage bias (ENC) and $w R_{N}$ is also not found in 432 random sequences with the same $\mathrm{CG}$ content as the rodent genes $(r=0.01, p=0.79)\left(\omega_{\mathrm{AA} / \mathrm{STOP}}=-10 ; \mathrm{CG} /\right.$ AT mutation bias $=0.9$; no transition/transversion bias). For the real 432 mouse (or rat) genes, there is instead a positive correlation (Fig. 7, Table 3b) be- 
Table 4. Correlation between variance of MD values for each amino acid and its contribution to codon usage bias

\begin{tabular}{|c|c|c|c|c|c|}
\hline \multirow[b]{2}{*}{$\operatorname{Bias}^{\mathrm{a}}$} & \multirow[b]{2}{*}{$\omega_{\mathrm{AA} / \mathrm{STOP}}$} & \multicolumn{2}{|c|}{ Twofold degenerate amino acids included } & \multicolumn{2}{|c|}{ Twofold degenerate amino acids excluded } \\
\hline & & $\chi^{\mathrm{b}}=0.9$ & $\chi=1$ & $\chi=0.9$ & $\chi=1$ \\
\hline \multicolumn{6}{|l|}{ No } \\
\hline & 0 & 0.42 & 0.47 & $0.81^{* * *}$ & $0.86^{* * * *}$ \\
\hline & -10 & $0.47^{*}$ & 0.46 & $0.83^{* * * *}$ & $0.78^{*}$ \\
\hline & -50 & $0.53 *$ & 0.41 & $0.85^{* * *}$ & 0.71 * \\
\hline \multicolumn{6}{|l|}{$\mathrm{C}$} \\
\hline & 0 & $0.52 *$ & 0.43 & $0.81 * *$ & $0.79 * *$ \\
\hline & -10 & $0.47^{*}$ & 0.41 & $0.83^{* * * *}$ & $0.72^{*}$ \\
\hline & -50 & $0.51 *$ & 0.40 & $0.84 * * *$ & $0.68^{*}$ \\
\hline
\end{tabular}

Note. Only degenerate amino acids are included. ${ }^{*} p<0.05 ;{ }^{*} p<0.01 ;{ }^{* *} p<0.005$ (the $p$ values differ because the number of amino acids on which the correlation is calculated is different).

${ }^{\mathrm{a}}$ Bias in the transition/transversion ratio: parameters as in Table 1.

${ }^{\mathrm{b}} \chi=\mathrm{CG} / \mathrm{AT}$ mutation ratio.

tween the degree of codon usage bias (ENC) and $w R_{N}$ (or $R_{N}$ ).

The result does not change drastically when "random" sequences with the position-specific CG content are used or when different values of $\omega_{\mathrm{AA} / \mathrm{STOP}}$ or for the transition /transversion ratio are considered. When only one or two mutation events are considered, the correlation with ENC still holds (Table 3).

The existence of a positive correlation between ENC and $w R_{N}$ means that as codon usage bias increases, the preference for optimal codons increases too. This suggests that codon usage bias is due, at least in part, to selection for error minimization at the protein level.

It may be thought that the difference between the random sequences and the real sequences is that the random sequences have no codon usage bias, and that error minimization is intrinsic in codon usage bias. To investigate this possibility, I also analyze sequences ( 82 for Drosophila, 432 for rodents-I use the mouse sequences, but results are virtually identical for rat) in which the frequency of synonymous codons is switched at random. In this case ENC values are exactly the same as in the real genes, but the relative frequencies of synonymous codons is modified.

If ENC was intrinsic in the measure of $w R_{N}$ (that is, if biased $w R_{N}$ values were simply due to biased codon usage), then the distribution of $w R_{N}$ (or $R_{N}$ ) values for these sequences would be similar to the distribution of $w R_{N}$ (or $R_{N}$ ) values of the real genes. On the contrary, the distribution of $w R_{N}$ values for these sequences with rearranged codon frequencies is not biased toward error minimization (it is similar to a Gaussian distribution; not shown), and ENC is not correlated with the degree of error minimization (Drosophila: $r=0.03, p=0.75$, for $w R_{N} ; r=-0.01, p=0.95$, for $R_{N}$; mouse: $r=-0.04, p=0.42$, for $w R_{N}$; $r=0.01, p=0.84$, for $\left.R_{N}\right)$.

\section{Error Minimization and Rates of Nucleotide Substitution}

If error minimization plays a role in the evolution of coding sequences, then the rate of nonsynonymous substitutions $\left(K_{a}\right)$ could be correlated with the degree of error minimization $\left(w R_{N}\right.$ or $\left.R_{N}\right)$, while this would not necessarily be the case for the rate of synonymous substitution $\left(K_{S}\right)$, because selection for error minimization at the protein level would occur only in the event of a nonsynonymous substitution. This means, in other words, that highly conserved genes are expected to prefer strongly codons that minimize the impact of errors due to mutation or mistranslation, while poorly conserved genes are expected to have more relaxed preferences.

I analyzed 38 of the Drosophila genes used for the previous analysis (the ones for which $K_{a}$ and $K_{s}$ measures were available - see Appendix) and found a positive correlation between $R_{N}$ or $w R_{N}$ values (Fig. 8) and the rates of nucleotide substitution, both synonymous and nonsynonymous. Changing the parameters does not change the results drastically (Table 3a). However, when only one mutation event is considered, the correlation with $K_{s}$ disappears. Note that ENC is correlated with $K_{s}$ $(r=0.69, p<0.0001)$ but not with $K_{a}(r=0.10$, $p=0.55)$ and that $K_{a}$ and $K_{s}$ are not correlated $(r=0.19, p=0.24)$.

The observed correlation with $K_{s}$ in Drosophila is rather unexpected. In rodents, however, for the 432 real genes analyzed here, $K_{a}$ and $w R_{N}$ are correlated but $K_{S}$ and $w R_{N}$ are not (for both mouse and rat; see Fig. 9 and Table $3 b$ ); even when $K_{4}$ is used instead of $K_{s}$ there is no correlation with $w R_{N}$ (for both mouse and rat, $r=-0.01, p=0.91)$. Different values for the transition/transversion bias or for the $\omega_{\mathrm{AA} / \mathrm{STOP}}$ score have negligible effects on the results (Table $3 b$ ). When only one or two mutation events are consid- 


\section{D. melanogaster}

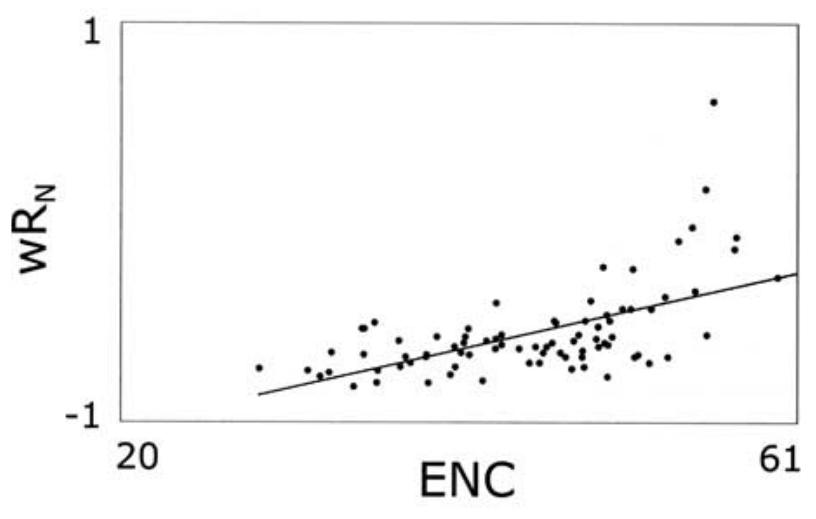

M. musculus

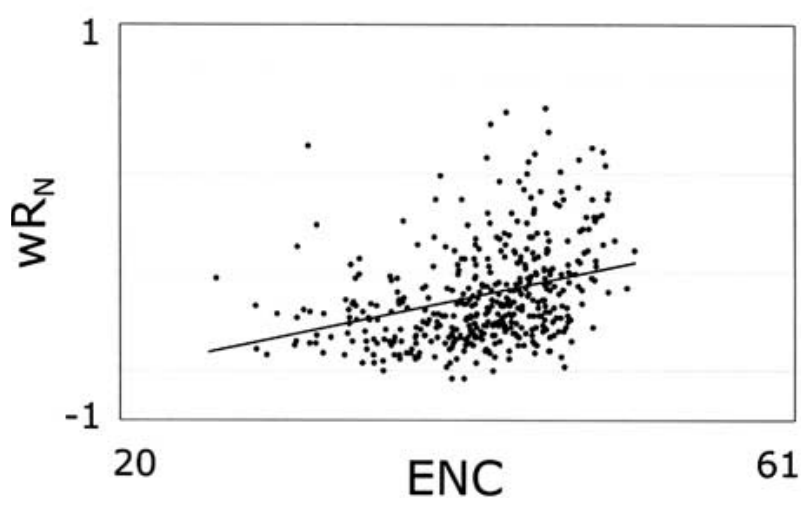

Fig. 7. $w R_{N}$ plotted against ENC for the 82 Drosophila and for the 432 mouse genes analyzed. The linear regression line is shown. $\omega_{\mathrm{AA} / \mathrm{STOP}}=-10$; no transition/transversion bias; CG/AT mutation ratio $=0.9$.

ered, the correlation with $K_{a}$ still holds, though with only one mutation it is weaker (Table $3 b$ ).

These results suggest that error minimization at the protein level plays a role in the evolution of coding sequences. An analysis of the single genes reveals that, for example, actin genes (known to be among the most conserved genes because of their fundamental role in the cytoskeleton and in the processes of muscle motility) have some of the lowest $w R_{N}$ values (for example, $w R_{N}=-0.79$ for the mouse $\alpha 1$-actin); the highest $w R_{N}$ value in mouse, on the contrary, is scored by the ST2 gene $\left(w R_{N}=+0.58\right)$, which has a high similarity to the interleukin-1 receptor, known to be among the most variable genes.

\section{Importance of Amino Acid Similarity Matrices}

For all the previous analyses I have used an amino acid similarity matrix based on chemical properties (McLachlan 1971). However, using different matrices may lead to very different results when analyzing the level of error minimization of the genetic code (Haig
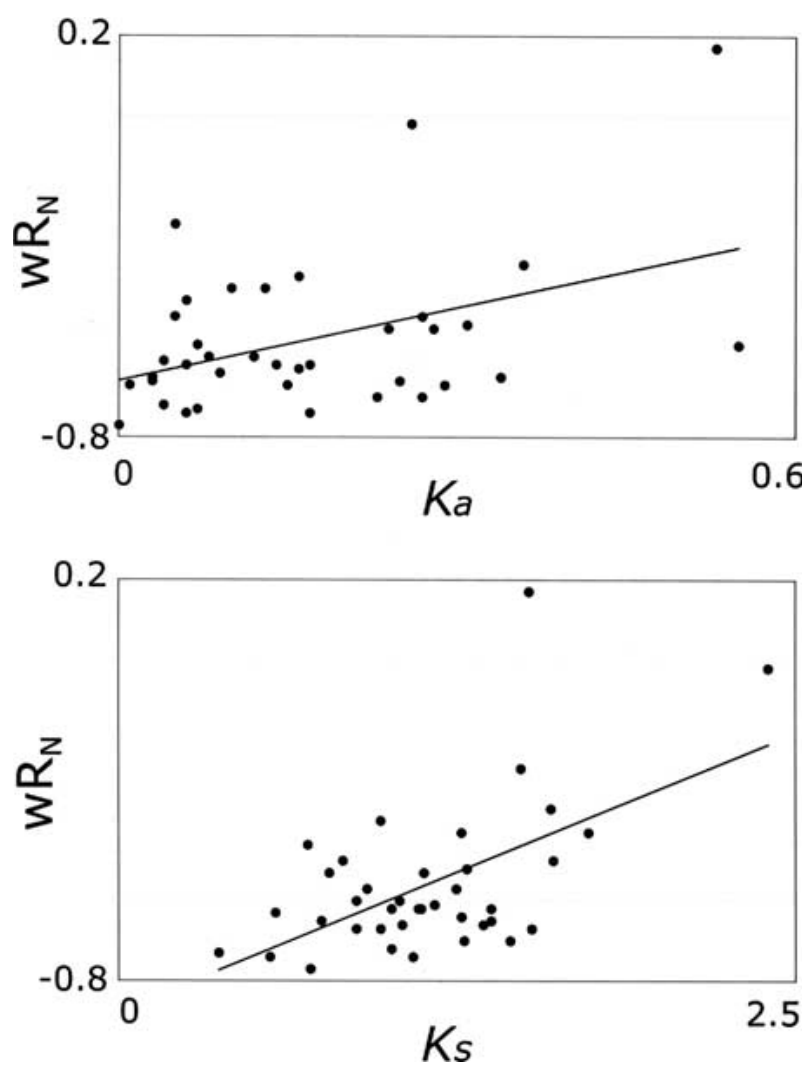

Fig. 8. $w R_{N}$ plotted against $K_{a}$ and $K_{s}$ for the 38 Drosophila genes analyzed. The linear regression line is shown. $\omega_{\mathrm{AA} / \mathrm{STOP}}=-10$; no transition/transversion bias; CG/AT mutation ratio $=0.9$.

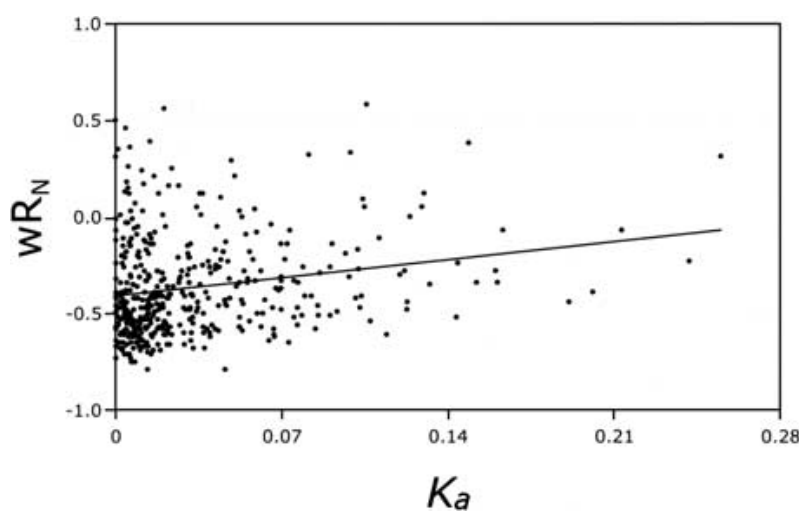

Fig. 9. $w R_{N}$ against $K_{a}$ for the 432 mouse genes analyzed. The linear regression line is shown. $\omega_{\mathrm{AA} / \mathrm{STOP}}=-10$; no transition/ transversion bias; $\mathrm{CG} / \mathrm{AT}$ mutation ratio $=0.9$.

and Hurst 1991; Archetti 2004). Therefore, I have checked the robustness of the results obtained here with McLachlan's matrix, by using different similarity matrices under different sets of parameters.

The main results do not change (Table 5); the two main differences are a weak correlation between $w R_{N}$ and CG content when a matrix based on hydrophobicity is used and a slightly weaker correlation between $w R_{N}$ and $K_{a}$ when the PAM 74-100 matrix is used. All in all it seems that using different matrices does not affect the main results. 
Table 5. Correlation between $w R_{N}$ and CG content, ENC, $K_{a}$ and $K_{s}$ for the 432 mouse genes when different similarity matrices are used

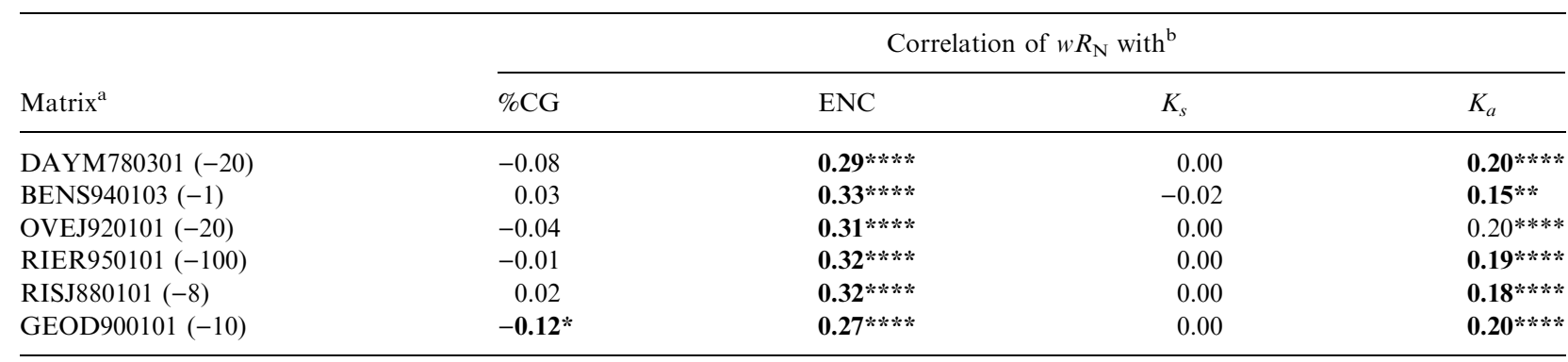

Note. Significant correlations are in boldface $(* p<0.05 ; * p<0.005 ; * * * p<0.0001)$.

${ }^{\mathrm{a}}$ The label corresponds to the AAindex accession number (http://www.genome.ad.jp/dbget/AAindex/list_of_matrices), followed (in parentheses) by the value of the similarity score with the stop codon $\left(\omega_{\mathrm{AA} / \mathrm{STOP}}\right)$ used here. DAYM780301: log-odds matrix for 250 PAMs (Dayhoff et al. 1978); BENS940103: log-odds scoring matrix collected in 74-100 PAM (Benner et al. 1994); OVEJ920101: STR matrix from structure-based alignments (Overington et al. 1992); RIER950101: hydrophobicity scoring matrix (Riek et al. 1995); RISJ880101: scoring matrix (Risler et al. 1988); GEOD900101: hydrophobicity scoring matrix (George et al. 1990).

${ }^{\mathrm{b}} \% \mathrm{CG}, \mathrm{CG}$ content; ENC, effective number of codons; $K_{s}$ and $K_{a}$, rates of synonymous and nonsynonymous substitution. CG/AT mutation ratio $=0.9$; no bias in the transition/transversion ratio; $\mathrm{MD}$ values calculated up to the 10th mutation event.

\section{Discussion}

The hypothesis presented here is based on the concept of error minimization at the protein level, a concept that has already been used to study the evolution of the genetic code. The idea discussed in this paper does not deal with the evolution of the genetic code itself but predicts that, given the genetic code (in which codons are arranged to minimize errors), within each synonymous family, the most used codons are the ones that, after mutation or mistranslation, keep on coding for the same amino acid or for amino acids with similar properties; that is, that codons are used in a way that minimizes errors.

To my knowledge this is the first time this hypothesis has been tested, though some hints in this direction have been put forward by Modiano et al. (1980) and McPherson (1988). McPherson (1988) suggested that codon preference may reflect mistranslational constraints, while Modiano et al. (1980) observed that codons that can mutate to termination codons ("pretermination" codons) by point mutation are avoided in human $\alpha$ and $\beta$-globin genes and suggested that it be so to avoid mutations with drastic effects. This hypothesis, however, has not received much attention. In his book on the neutral theory of natural selection, Kimura (1983) mentions it only to dismiss it in few words: "It seems to me that the selective advantage coming from such strategy [avoiding pre-termination codons] is too small (presumably the order of the mutation rate) to be effective in the actual course of evolution." However, the work eventually done on codon usage evolution has shown that selection is in fact able to operate at a very fine scale; as Duret (2002) puts it, selection is able to act on codon usage even if it leads to a synonymous substitution on a single gene in a whole genome. Moreover, if selection shaped the genetic code, it is not inconceivable that it also shaped codon usage, though selection for codon usage may have occurred after a genetic code had been fixed (or perhaps coevolved).

I have developed a theoretical measure of the capacity of each codon to minimize the effects of errors due to mutation and mistranslation. I have then used these values to measure the degree of error minimization for individual genes of Drosophila melanogaster and rodents.

The main result is (i) that the distribution of these values is not random: both Drosophila melanogaster and rodents prefer codons that lead to the minimization of errors at the protein level, that is, codons that, after mutation or mistranslation, keep on coding for the same amino acid or for amino acids with similar properties. Therefore, error minimization at the protein level may affect not only the evolution of the genetic code itself, as has long been discussed, but also the frequency of synonymous codons.

I have also shown (ii) that it is possible to exclude that GC content is solely responsible for the pattern observed, as the degree of error minimization is not correlated with CG content, and random sequences with the same CG content do not lead to error minimization. This suggests that selection, and not mutation bias, is responsible for the observed pattern of error minimization.

I have then shown (iii) that the higher the bias in codon usage, the stronger the tendency to use codons that minimize errors, and that (iv) the higher the contribution of an amino acid to codon usage bias, the higher the variance of the capacity to minimize errors for its synonymous codons. This suggests that codon usage bias can be explained by the preferential usage of codons that minimize errors. Other evidence (McVean and Vieira 2001) suggests that Leu (the amino acid shown here to have the highest variance 
for MD values) is the amino acid on which the influence of selection is strongest.

Classically, two models have been proposed to explain codon usage bias: the selective model, which postulates that there is a coadaptation of codon usage and tRNA abundance; and the neutral model, which postulates that the bias results from biases in the mutational process (see recent review by Duret 2002). In unicellular organisms, codon usage bias is usually thought to be due to selection for translation efficiency: there is preferential usage of codons that bind more efficiently to the corresponding tRNA or that are recognized by a more abundant tRNA (Post et al. 1979; Ikemura 1981, 1982, 1985, 1992). In this way proteins are produced more rapidly and with fewer errors. In mammals codon usage may be related to isochores, segments several kilobases long of similar CG content but with substantial differences in CG content between them (Bernardi et al. 1985). In Drosophila it has been suggested that codon usage bias is caused by selection on synonymous sites for translational accuracy (Akashi 1994; Powell and Moriyama 1997), but there is evidence that other factors, for example, recombination and compositional bias, influence codon usage in Drosophila (Marais et al. 2001; Hey and Kliman 2002). In general, therefore, it is thought that codon usage reflects a combination of different forces. The results presented here show that it is possible that selection on error minimization plays a role also in the evolution of codon usage bias.

The results seem rather robust, not depending drastically on the transition/transversion ratio or on the CG/AT mutation ratio. I have also shown that they do not depend on the amino acid similarity matrix used. Matrices based on observed substitutions are usually considered better than chemical similarity matrices, but they are derived from sequences that include themselves codon usage bias; for this reason these matrices may not provide a fair measure (though it is not demonstrated that this is the case) to study the relationship between error minimization and codon usage bias. Di Giulio (2001) used a similar argument to critique the use of the PAM 74-100 matrix in studies of the genetic code origin. Note, however, that there is a difference: in the study of the origin of the genetic code, the similarity matrix used must be independent from the structure of the genetic code, otherwise the analysis would be tautologous (genetic code structure explained with a matrix that depends on the genetic code structure). In this case, instead, even if the similarity matrix depended on the genetic code structure, this would not affect the analysis; indeed the hypothesis I want to test is that codon usage depends on the similarity matrix and on the arrangement of codons in the genetic code (codon usage bias explained with a matrix that may depend on the genetic code structure).
Error minimization seems to be weaker when only one mutation is considered instead of multiple mutation events. This might suggest that mutation is more important than mistranslation in natural selection for the minimization of errors at the protein level. If selection during mistranslation is important, there might be a correlation between error minimization and gene length or gene expression. Studies on the evolution of the genetic code have been rather vague as to whether the main mechanism of selection for error minimization acts during translation or mutation. The analysis presented here also does not lead to a conclusive answer concerning the relative importance of mutation and mistranslation for error minimization due to codon usage. The observation that error minimization is stronger with multiple mutation events and other preliminary results of mine (M.A., unpublished) suggest that mutation rather than mistranslation is the driving force of error minimization at the protein level. If mistranslation is important in selection for error minimization, then gene expression levels should be correlated with the degree of error minimization, as highly expressed genes should be the ones under stronger selective pressure to reduce errors. This is a point that would be worth investigating.

Finally I have shown (v) that the degree of error minimization varies according to the rate of evolution of the gene. That is, genes with a low rate of evolution tend to use more codons that minimize the effect of errors. There is, however, a difference between rodents and Drosophila. In rodents only the rate of nonsynonymous substitution is correlated with the degree of error minimization, while in Drosophila the rate of synonymous substitution is also correlated with the degree of error minimization. Possibly this difference is due to the different methods used to calculate the substitution rates. For the 432 rodent genes used here, $K_{a}$ and $K_{s}$ have been obtained by Smith and Hurst (1997) by a maximum-likelihood approach (ML), while for the Drosophila genes $K_{a}$ and $K_{s}$ had been obtained by Akashi (1994) using the approximate method of Nei and Gojobori (1986) (NG). It has been shown (Yang and Nielsen 1998, 2000; Bielawski et al. 2000) that ML methods are superior to approximate methods and that the NG method may lead to biased estimates of substitution rates. It is possible that the correlation between $w R_{N}$ and $K_{s}$ observed in Drosophila is due to the usage of approximate methods in the calculation of the substitution rates. It seems that it cannot be just an artifact of a correlation of $K_{s}$ with $K_{a}$, because in that case (Akashi 1994) $K_{s}$ and $K_{a}$ are not correlated. The alternative possibility is that $w R_{N}$ and $K_{s}$ are correlated in Drosophila but not in mammals. To decide about these two alternatives a reexamination of Drosophila genes, with $K_{s}$ and $K_{a}$, derived by ML methods would be useful. 
What can we deduce from this correlation? Highly conserved genes are expected to have a high degree of error minimization if the minimization of errors at the protein level is important, because any change in these genes would result in a change in the function of the protein and, as a consequence, if the change is deleterious, a reduction in fitness. Less conserved genes, instead, can have more relaxed preferences on codon usage because changes at the protein level will not have a drastic impact on fitness. On the other hand, it could be possible to argue that genes evolving faster should show error minimization even more clearly, because they are more likely to have evolved to their current state by means of small mutations. This is a basic question in molecular evolution. Amino acid replacement changes should be expected to have greater influence on genes whose structure and function is "important." Unfortunately, as Kreitman (1996) points out, the problem is not so simple: constraining selection alone cannot explain protein evolution because the observed differences in proteins must, by definition, be ones that have escaped constraining selection.
The study presented here does not help understanding this problem, but further studies on error minimization might provide some useful insights. The method itself could be used to detect selection from single DNA sequences.

The degree of error minimization could also depend on gene function. Genes of parasitic organisms that code for proteins involved in host-parasite interactions, for example, in some cases prefer codons that increase the effect of errors to escape the host defenses more easily (M.A., upublished). This might lead to different optimal codons for different species, according to their life history.

Indeed, the explanation of codon usage bias presented here, based on the structure of the genetic code, should predict a unique set of optimal codons for all the species that use the same code. I have shown here that results for Drosophila are consistent with those for rodents. A comprehensive analysis of many more species will be presented in another paper, with a more precise method to detect selection from codon usage analysis of single nucleotide sequences (M.A., unpublished).

\section{Appendix}

Table AI. $w R_{N}$ values for different values of the parameters for 82 Drosophila melanogaster genes

\begin{tabular}{|c|c|c|c|c|c|c|c|}
\hline Gene & $w R_{N-0}$ & $w R_{N-10}$ & $w R_{N-20}$ & $w R_{N-50}$ & $w R_{N-10(A)}$ & $w R_{N-10(B)}$ & $w R_{N-10(C)}$ \\
\hline *ade3 & -0.42 & -0.43 & -0.46 & -0.43 & -0.38 & -0.51 & -0.49 \\
\hline${ }^{*} A d h$ & -0.67 & -0.74 & -0.73 & -0.70 & -0.61 & -0.70 & -0.72 \\
\hline${ }^{*} A d h r$ & -0.24 & -0.27 & -0.25 & -0.21 & -0.09 & -0.27 & -0.27 \\
\hline${ }^{*} A m y-d$ & -0.73 & -0.73 & -0.69 & -0.65 & -0.62 & -0.78 & -0.73 \\
\hline${ }^{*}$ Antp & -0.76 & -0.72 & -0.68 & -0.64 & -0.62 & -0.81 & -0.75 \\
\hline${ }^{*}$ Aprt & -0.55 & -0.64 & -0.62 & -0.60 & -0.49 & -0.63 & -0.63 \\
\hline${ }^{*} b c d$ & -0.73 & -0.60 & -0.54 & -0.49 & -0.56 & -0.74 & -0.65 \\
\hline *boss & -0.42 & -0.43 & -0.41 & -0.38 & -0.37 & -0.43 & -0.43 \\
\hline$b w$ & -0.80 & -0.76 & -0.70 & -0.65 & -0.68 & -0.86 & -0.78 \\
\hline$c d c 37$ & -0.77 & -0.79 & -0.77 & -0.74 & -0.72 & -0.81 & -0.79 \\
\hline${ }^{*} C p 15$ & -0.37 & -0.57 & -0.60 & -0.61 & -0.43 & -0.49 & -0.54 \\
\hline${ }^{*} C p 16$ & -0.44 & -0.53 & -0.55 & -0.55 & -0.47 & -0.46 & -0.50 \\
\hline${ }^{*}$ Cp 18 & -0.38 & -0.50 & -0.51 & -0.50 & -0.44 & -0.43 & -0.46 \\
\hline${ }^{*}$ Cp 19 & -0.49 & -0.65 & -0.66 & -0.65 & -0.55 & -0.60 & -0.63 \\
\hline${ }^{*} \mathrm{Cp} 36$ & -0.58 & -0.63 & -0.60 & -0.56 & -0.56 & -0.63 & -0.63 \\
\hline$c s w$ & -0.77 & -0.63 & -0.62 & -0.57 & -0.60 & -0.82 & -0.66 \\
\hline$c y b t-b 5$ & -0.48 & -0.66 & -0.50 & -0.48 & -0.51 & -0.54 & -0.53 \\
\hline$D d x 1$ & -0.74 & -0.77 & -0.74 & -0.71 & -0.65 & -0.79 & -0.77 \\
\hline$d p p$ & -0.76 & -0.70 & -0.63 & -0.56 & -0.58 & -0.83 & -0.74 \\
\hline$e(r)$ & -0.57 & -0.59 & -0.56 & -0.53 & -0.51 & -0.63 & -0.61 \\
\hline *elav & -0.75 & -0.65 & -0.57 & -0.50 & -0.57 & -0.77 & -0.68 \\
\hline "en & -0.60 & -0.62 & -0.59 & -0.55 & -0.53 & -0.70 & -0.64 \\
\hline esc & -0.61 & -0.59 & -0.55 & -0.52 & -0.51 & -0.67 & -0.61 \\
\hline${ }^{*}$ Est-6 & 0.01 & -0.02 & -0.02 & 0.00 & 0.05 & -0.04 & -0.03 \\
\hline exu & -0.50 & -0.49 & -0.46 & -0.43 & -0.46 & -0.51 & -0.48 \\
\hline$F b p 2$ & -0.75 & -0.80 & -0.78 & -0.75 & -0.62 & -0.82 & -0.80 \\
\hline${ }^{*}$ Fmrf & -0.55 & -0.53 & -0.50 & -0.47 & -0.51 & -0.59 & -0.55 \\
\hline$f u$ & -0.79 & -0.73 & -0.68 & -0.63 & -0.67 & -0.82 & -0.75 \\
\hline$f z$ & -0.72 & -0.67 & -0.62 & -0.57 & -0.52 & -0.74 & -0.69 \\
\hline$g l$ & -0.65 & -0.65 & -0.60 & -0.56 & -0.54 & -0.75 & -0.67 \\
\hline Gld & -0.62 & -0.62 & -0.58 & -0.53 & -0.54 & -0.65 & -0.63 \\
\hline${ }^{*} G p d h$ & -0.66 & -0.66 & -0.63 & -0.60 & -0.58 & -0.67 & -0.66 \\
\hline${ }^{*} h$ & -0.58 & -0.60 & -0.58 & -0.55 & -0.51 & -0.66 & $\begin{array}{l}-0.61 \\
\text { continued }\end{array}$ \\
\hline
\end{tabular}


Table AI. Continued

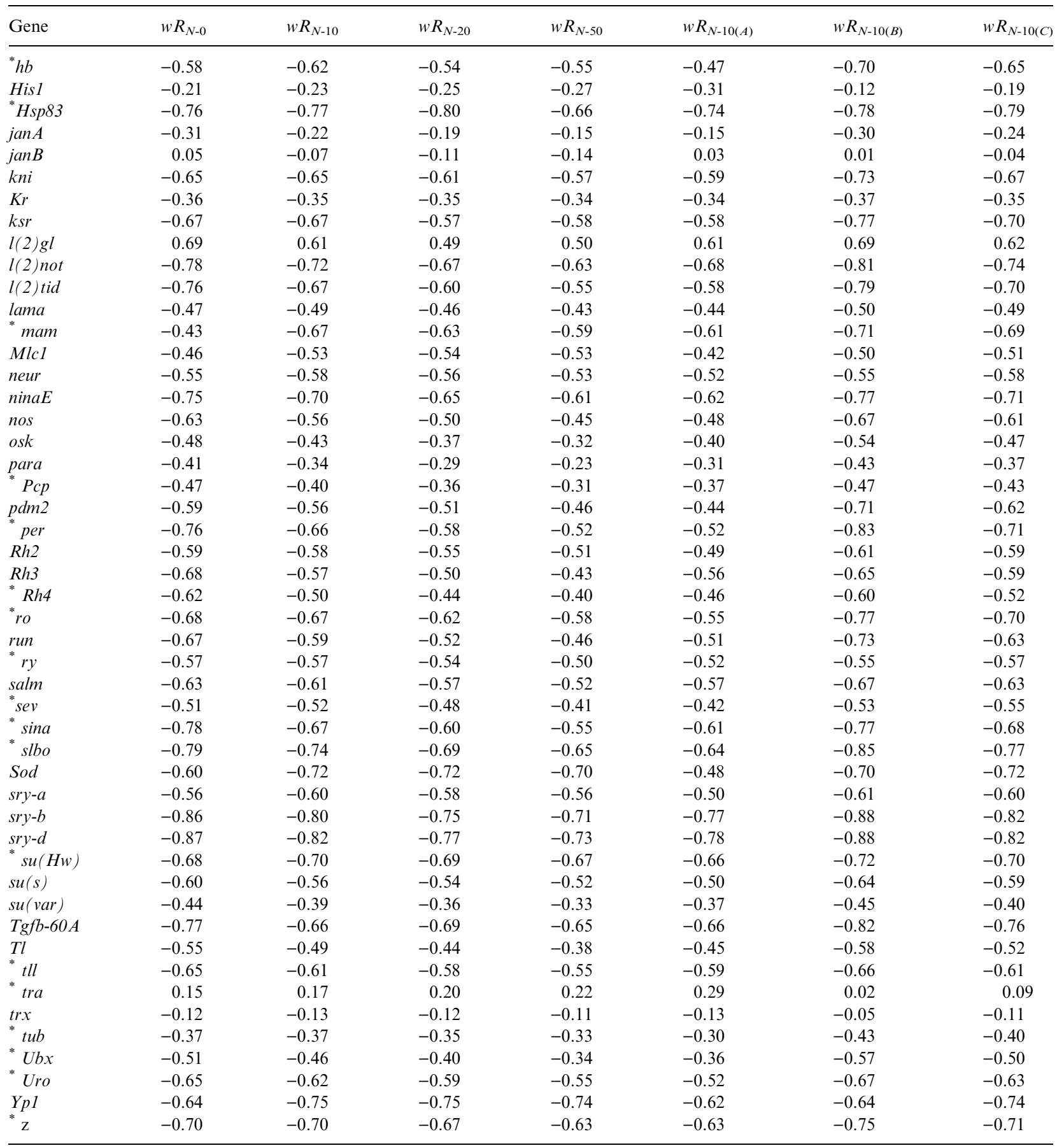

Note. $w R_{N-X(Y)}$ is the $w R_{N}$ value calculated with $\omega_{\mathrm{AA} / \mathrm{STOP}}=X$ and with values of the transition/transversion ratio corresponding to $Y$ (see Table 1). Accession numbers for all the genes are given by Moriyama and Powell (1997). An asterisk precedes genes used in the analysis of correlation with $K_{s}$ and $K_{a}$ (taken from Akashi 1994).

Acknowledgments. Nick Smith provided the substitution rates used by Smith and Hurst (1997).

\section{References}

Akashi H (1994) Synonymous codon usage in Drosophila melanogaster: Natural selection and translational accuracy. Genetics 136:927-935
Archetti M (2004) Codon usage bias and mutation constraints reduce the level of error minimization of the genetic code. J Mol Evol (in press)

Benner SA, Cohen MA, Gonnet GH (1994) Amino acid substitution during functionally constrained divergent evolution of protein sequences. Protein Eng 7(11):1323-1332

Bernardi G, Olofsson B, Filipski J, Zerial M, Salinas J, Cuny G, Meunier-Rotival M, Rodier F (1985) The mosaic genome of warm-blooded vertebrates. Science 228:953-958 
Bielawski JP, Dunn KA, Yang Z (2000) Rates of nucleotide substitution and mammalian nuclear gene evolution: approximate and maximum-likelihood methods lead to different conclusions. Genetics 156:1299-1308

Dayhoff MO, Schwartz RM, Orcutt BC (1978) A model of evolutionary change in proteins.. In: Dayhoff MO (ed) Atlas of protein sequence and structure, Vol 5, Supp1 3. National Biomedical Research Foundation, Washington, DC, p 352

Di Giulio M (2000a) Genetic code origin and the strength of natural selection. J Theor Biol 205:659-661

Di Giulio M (2000b) The origin of the genetic code. Trends Biochem Sci 25(2):44

Di Giulio M (2001) The origin of the genetic code cannot be studied using measurements based on the PAM matrix because this matrix reflects the code itself, making any such analyses tautologous. J Theor Biol 208:141-144

Duret L (2002) Evolution of synonymous codon usage in metazoans. Curr Opin Gen Dev 12:640-649

Epstein CJ (1966) Role of the amino-acid "code" and of selection for conformation in the evolution of proteins. Nature 210:25-28

Freeland SJ, Hurst LD (1998) The genetic code is one in a million. J Mol Evol 47:238-248

Freeland SJ, Knight RD, Landweber LF (2000) Measuring adaptation within the genetic code. Trends Biochem Sci 25(2):44-45

George DG, Barker WC, Hunt LT (1990) Mutation data matrix and its uses. Methods Enzymol 183:333-351

Grantham R, Gautier C, Gouy M, Mercier R, Pavè A (1980) Codon catalog usage and the genome hypothesis. Nucleic Acid Res 8:r49-r62

Haig D, Hurst LD (1991) A quantitative measure of error minimization in the genetic code. J Mol Evol 33(5):412-417

Hey J, Kliman RM (2002) Interactions between natural selection, recombination and gene density in the genes of Drosopila. Genetics 160:595-608

Ikemura T (1981) Correlation between the abundance of Escherichia coli transfer RNAs and the occurrence of the respective codons in its protein genes: a proposal for a synonymous codon choice that is optimal for the E. coli translational system. J Mol Biol 151:389-409

Ikemura T (1982) Correlation between the abundance of yeast transfer RNAs and the occurrence of the respective codons in protein genes Differences in synonymous codon choice patterns of yeast and Escherichia coli with reference to the abundance of isoaccepting transfer RNAs. J Mol Biol 158:573-597

Ikemura T (1985) Codon usage and tRNA content in unicellular and multicellular organisms. Mol Biol Evol 2:13-34

Ikemura T (1992) Correlation between codon usage and tRNA content in microorganisms. In: Hatfield DL, Lee BJ, Pirtle RM (eds) Transfer RNA in protein synthesis. CRC Press, Boca Raton, pp 87-111

Kimura M (1983) The Neutral Theory of Natural Selection. Cambridge University Press, Cambridge

Knight RD, Freeland SJ, Landweber LF (1999) Selection, history and chemistry: The three faces of the genetic code. Trends Biochem Sci 24(6):241-247

Kreitman M (1996) The neutral theory is dead. Long live the neutral theory. BioEssays 18:678-682
Marais G, Mouchiroud D, Duret L (2001) Does recombination improve selection on codon usage? Lessons from nematode and fly complete genomes. Proc Natl Acad Sci USA 98:5688-5692

McLachlan AD (1971) Tests for comparing related amino-acid sequences cytochrome c and cytochrome c 551. J Mol Biol 61:409424

McPherson DT (1988) Codon preference reflects mistranslational constraints: A proposal. Nucleic Acid Res 16:4111-4120

McVean GAT, Vieira J (2001) Inferring parameters of mutation, selection and demography from patterns of synonymous site evolution in Drosophila. Genetics 157(1):245-257

Modiano G, Battistuzzi G, Motulsky AG (1981) Nonrandom patterns of codon usage and of nucleotide substitutions in human alpha- and beta-globin genes: an evolutionary strategy reducing the rate of mutations with drastic effects? Proc Natl Acad Sci USA 78:1110-1114

Moriyama EN, Powell JR (1997) Codon usage bias and tRNA abundance in Drosophila. J Mol Evol 45:514-523

Nakamura Y, Gojobori T, Ikemura T (2000) Codon usage tabulated from the the international DNA sequence databases: Status for the year 2000. Nucleic Acids Res 28:292

Nei M, Gojobori T (1986) Simple methods for estimating the number of synonymous and non-synonymous nucleotide substitution. Mol Biol Evol 3:418-426

Overington J, Donnelly D, Johnson MS, et al. (1992) Environmentspecific amino-acid substitution tables-Tertiary templates and prediction of protein folds. Protein Sci 1(2):216-226

Post LE, Strycharz GD, Nomura M, Lewis H, Dennis PP (1979) Nucleotide sequence of the ribosomal protein gene cluster adjacent to the gene for RNA polymerase subunit beta in Escherichia coli. Proc Natl Acad Sci USA 76:1697-1701

Powell JR, Moriyama EN (1997) Evolution of codon usage bias in Drosophila. Proc Natl Acad Sci USA 94:7784-7790

Riek RP, Handschumacher MD, Sung SS, Tan M, Glynias MJ, Schluchter MD, Novotny J, Graham RM (1995) Evolutionary conservation of both the hydrophilic and hydrophobic nature of transmembrane residues. J Theor Biol 172(3):245258

Risler JL, Delorme MO, Delacroix H, Henaut A (1988) Amino acid substitutions in structurally related proteins. A pattern recognition approach. Determination of a new and efficient scoring matrix. J Mol Biol 204(4):1019-1029

Smith NG, Hurst LD (1997) The effect of tandem substitutions on the correlation between synonymous and non-synonymous rates in rodents. Genetics 153:1395-1402

Topal MD, Fresco JR (1976) Complementary base pairing and the origin of substitution mutations. Nature 263:285-289

Woese CR (1965) On the evolution of the genetic code. Proc Natl Acad Sci USA 54:1546-1552

Wright F (1990) The 'effective number of codons' used in a gene. Gene 87:23-29

Yang Z, Nielsen R (2000) Estimating synonymous and non-synonymous rate variation in nuclear genes of mammals. $\mathrm{J} \mathrm{Mol}$ Evol 46:409-418

Yang Z, Nielsen R (1998) Synonymous and non-synonymous substitution rates under realistic evolutionary models. Mol Biol Evol 17(1):32-43 